\title{
Paraplegia after open surgery using endovascular stent graft for aortic arch aneurysm
}

Takeshi Miyairi, MD, ${ }^{a}$ Yutaka Kotsuka, MD, ${ }^{a}$ Tetsuro Morota, MD, ${ }^{a}$ Hiroshi Kubota, MD, ${ }^{a}$ Ko Shibata, MD, ${ }^{a}$ Yorinobu Ikeda, MD, ${ }^{a}$ Tadashi Kitamura, MD, ${ }^{a}$ Takeshi Kashima, ${ }^{b}$ and Shinichi Takamoto, ${ }^{a}$ MD, Tokyo, Japan

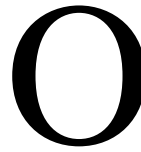

pen surgery with an endovascular stent graft is a novel therapeutic technique for aortic aneurysm that is especially useful for aneurysms of the distal arch. In this report we describe the clinical course and autopsy find-

From the Departments of Cardiothoracic Surgery a and Human Pathology, ${ }^{\mathrm{b}}$ University of Tokyo, Tokyo, Japan.

Received for publication Feb 28, 2001; accepted for publication April 19, 2001.

Address for reprints: Takeshi Miyairi, $\mathrm{MD}, \mathrm{PhD}$, Department of Cardiothoracic Surgery, University of Tokyo, Hongo 7-3-1, Bunkyo-ku, 1138655 Tokyo, Japan (E-mail: tmiyairi-tky@umin.ac.jp).

J Thorac Cardiovasc Surg 2001;122:1240-3

Copyright (C) 2001 by The American Association for Thoracic Surgery

$0022-5223 / 2001 \$ 35.00+0 \quad \mathbf{1 2 / 5 4 / 1 1 6 9 3 7}$

doi:10.1067/mtc.2001.116937 ings of 2 patients who had paraplegia after undergoing this procedure and died during the hospital stay.

\section{Clinical Summary}

PATIENT 1. A 78-year-old man had angina pectoris and intermittent claudication. Angiograms revealed a thoracic aortic aneurysm in the distal part of the aortic arch involving the left subclavian artery, severe stenosis in the right coronary and left circumflex arteries, and total occlusion of both superficial femoral arteries. After median sternotomy and establishment of cardiopulmonary bypass, bypass grafts to the 2 coronary arteries were performed during perfusion cooling. During hypothermic circulatory arrest at a tympanic temperature of $18^{\circ} \mathrm{C}$ and retrograde cerebral perfusion, the aortic arch was transected between the left common carotid artery and the left subclavian artery. A stent graft, which was constructed from a $40 \times 50$-mm selfexpandable Gianturco Z-shaped stent (William Cook Europe, Bjaeverskov, Denmark), covered with the distal part of a $32.5-\mathrm{mm}$ thin-walled woven Dacron graft, was delivered into the descending 

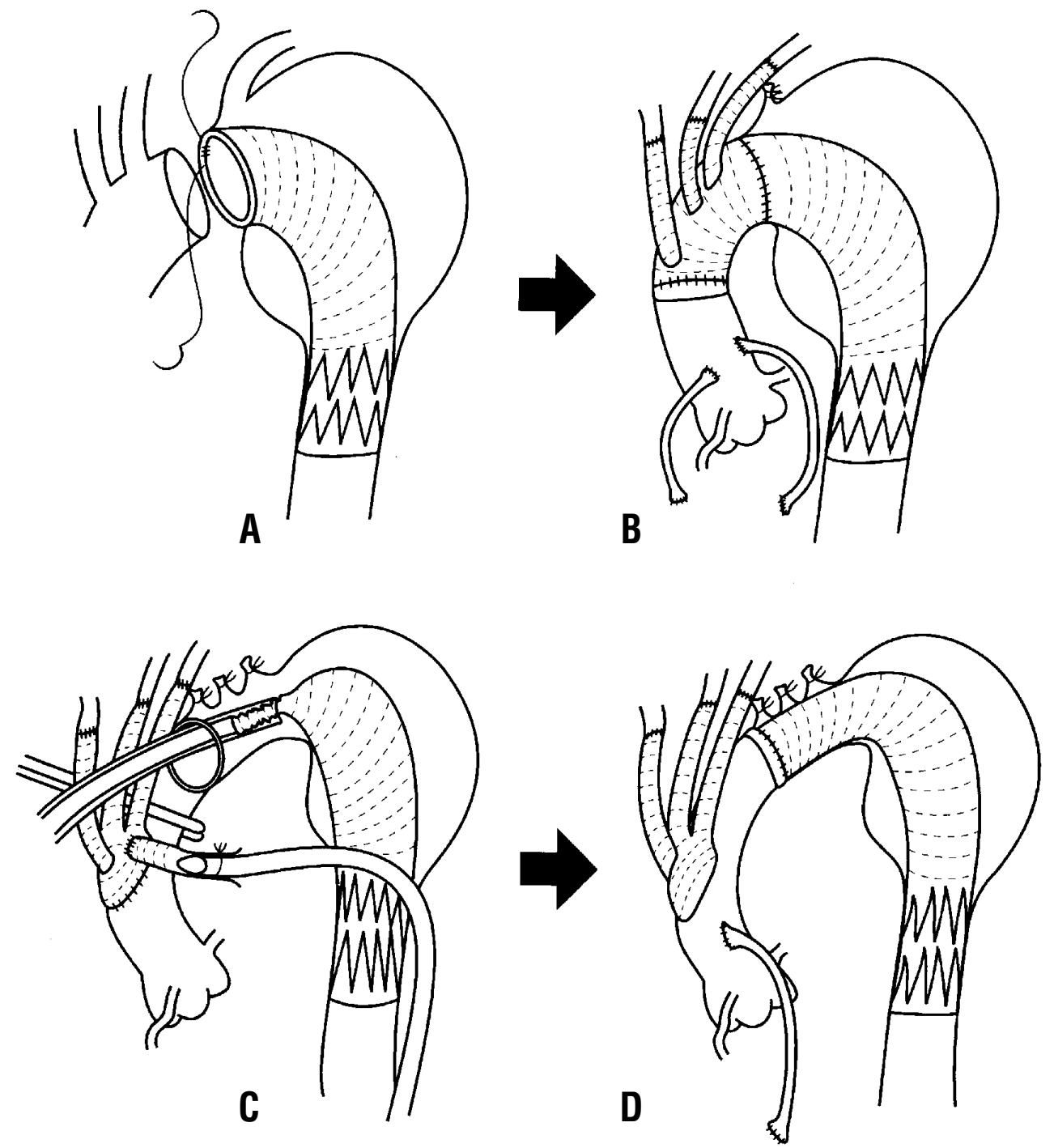

Figure 1. Schematic drawing of the operative procedure. A, The proximal end of the stent graft, which was inserted into the descending aorta, is sutured to the transected distal aorta. $B$, The branched graft is anastomosed to the proximal end of the stent graft and the native aorta. The 3 arch vessels are reconstructed with the branches of the graft. C, The 3 arch vessels are connected to the branches of the prosthesis that is attached to the ascending aorta. Then, the stent graft is delivered into the descending aorta by means of the sheath catheter. D, The proximal end of the stent graft is sutured to the aortic wall. A and B, Patient 1. C and D, Patient 2.

aorta by means of a 30F sheath catheter with the distal end located 5 $\mathrm{cm}$ below the aneurysm. The proximal end of the stent graft was sutured to the wall of the transected aortic arch (Figure 1, A). A 26mm collagen-coated woven Dacron graft with 4 branches was connected to the stent graft, and 3 arch vessels were reconstructed with the branches of the graft. Under systemic and hemi-selective cerebral perfusion through the fourth branch, the proximal anastomosis was completed (Figure 1, B) and the heart was reperfused. The cardiopulmonary bypass, aortic crossclamp, and circulatory arrest times were 317 minutes, 190 minutes, and 80 minutes, respectively.

A state of almost complete loss of sensory and motor function below the level of the sixth thoracic vertebrae existed throughout the postoperative course, and the patient died of pneumonia on the twenty-first postoperative day.

At autopsy, there were no abnormal findings in the placement of the stent graft, the anastomoses, or the reconstruction of the 3 arch vessels. The aneurysmal cavity was occluded with thrombus. The Adamkiewicz artery was confirmed as the ninth intercostal artery by its characteristic hairpin curve just before connecting with the anterior spinal artery (Figure 2,A). The orifice of the Adamkiewicz artery was located $1 \mathrm{~cm}$ below the distal end of the stent graft (Figure 2, B). Histologic examination revealed disappearance of neuronal cells and axonal swelling of the lateral and posterior segments of the spinal cord at the level of the fourth 

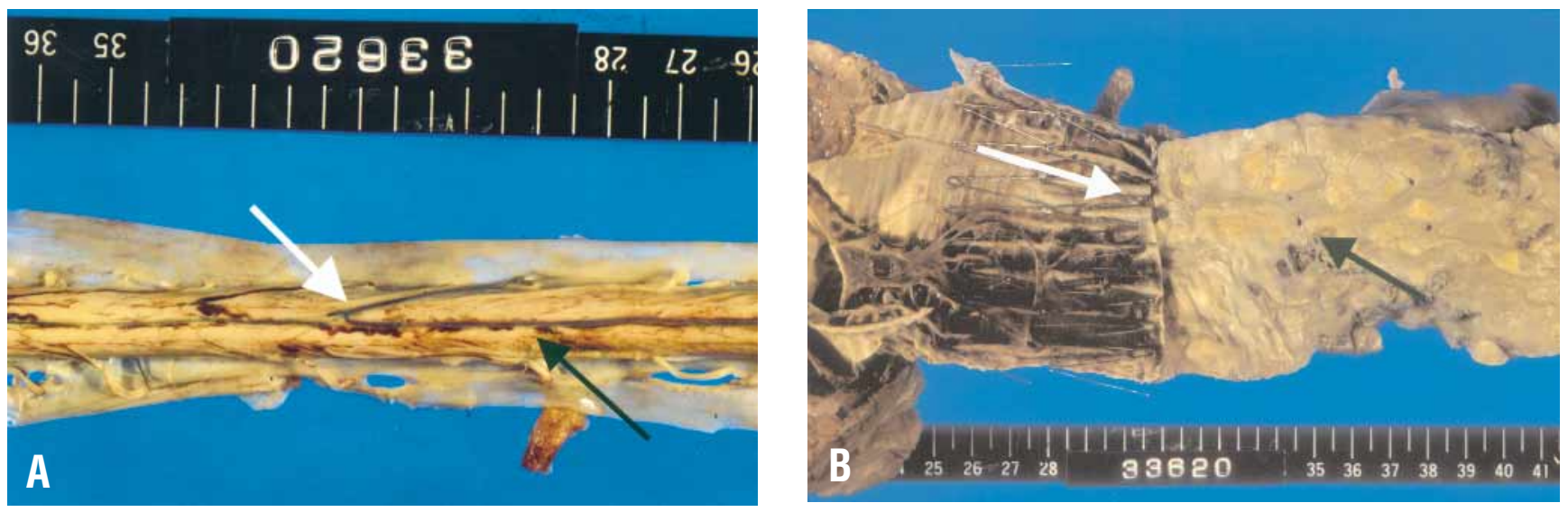

Figure 2. A, Photograph from case 1 (left is cranial side): The white arrow indicates the Adamkiewicz artery at the level of the ninth spinal anterior thoracic root, on the left side of the spinal cord. The Adamkiewicz artery is confirmed by its characteristic course of a hairpin bend just before joining the anterior spinal artery (black arrow). Indocyanine green solution was injected into the arteries. B, Photograph from case 1 (left is cranial side): The white arrow shows the distal end of the endovascular stent graft located at the level of the eighth spinal anterior thoracic root. The black arrow indicates the orifice of the Adamkiewicz artery at the level of the ninth spinal anterior thoracic root.

spinal anterior thoracic root. Neither thrombus nor embolus was observed in any of the arteries.

PATIENT 2. An 80-year-old man had a history of hypertension, repair for an abdominal aortic aneurysm 10 years before, and chronic renal failure with hemodialysis for 3 years. Angiograms revealed a thoracic aortic aneurysm in the distal arch just below the left subclavian artery, a thoracoabdominal aneurysm extending from $3 \mathrm{~cm}$ above the diaphragm to the level of the celiac artery, and severe stenosis in the left anterior descending coronary artery. First, during perfusion cooling, the 3 arch vessels were connected to the branches of the prosthesis that was attached to the ascending aorta, and coronary artery bypass grafting was performed. At a tympanic temperature of $22^{\circ} \mathrm{C}$, the ascending aorta was crossclamped distal to the attachment of the prosthesis, thus maintaining perfusion of the heart, the brain, and the upper half of the body while the lower half was in circulatory arrest. The anterior wall of the ascending aorta, $1 \mathrm{~cm}$ proximal to the right brachiocephalic artery, was incised, and a stent graft made from a $40 \times 50-\mathrm{mm} \mathrm{Z}$ stent and a $37.5-\mathrm{mm}$ woven Dacron graft was inserted in the same manner as for patient 1 (Figure 1,C). The proximal end of the stent graft was sutured inclusively to the posterior wall and exclusively to the anterior wall of the ascending aorta, and the aortotomy was closed (Figure 1,D). The cardiopulmonary bypass time and circulatory arrest time for the lower part of the body were 286 minutes and 67 minutes, respectively. Hemodynamics immediately after bypass were unstable, with an arterial blood pressure of about 80 $\mathrm{mm} \mathrm{Hg}$ for 2 hours.

Postoperatively, incomplete tetraplegia with loss of superficial and deep sensation below the level of the fourth thoracic vertebra and motor dysfunction below the seventh were observed. Cerebrospinal fluid drainage was performed for 6 days. Although motion of the upper extremities gradually improved, that of the lower extremities remained unchanged. The patient had postoperative mediastinitis and died on postoperative day 31 .
At autopsy, there appeared to be no problems in the placement and anastomosis of the stent graft, and all 3 arch vessels were patent. The aneurysm was completely occluded with thrombus. The Adamkiewicz artery was confirmed as the twelfth intercostal artery 4 $\mathrm{cm}$ below the stent graft. Histologic examination revealed diffuse spinal infarction in the central to the anterior segments of the spinal cord, all continuous from the level of C6 to Th11. Disappearance of neuronal cells and spongiosis with form cell infiltration was prominent. A few clusters of cholesterin embolus occluding arteries that drained into the anterior or posterior spinal artery at the level of C8 and Th6 were also observed (Figure 3).

\section{Discussion}

Open surgery with an endovascular stent graft used for aortic arch aneurysm, first reported by Kato and colleagues, ${ }^{1}$ has several advantages over conventional prosthetic replacement. Substitution of stentgraft placement for distal anastomosis substantially reduces operative time, bleeding, and injury to adjacent organs resulting from dissection. As compared with catheter-based stent grafting, this method is especially useful for those distal arch aneurysms adjacent to or even including the origin of the left subclavian artery, where proximal landing space is difficult to obtain. Since 1996, we ${ }^{2}$ have applied this method to 19 high-risk patients with distal aortic arch aneurysms with a fair hospital mortality (10.5\%). However, 4 patients had postoperative paraplegia, with 2 hospital deaths. Although the etiology of paraplegia resulting from this method is not yet clear, the autopsy findings of these 2 cases were very informative.

Blood supply from the Adamkiewicz artery, which perfuses the anterior two thirds of the spinal cord, is thought to be highly associated with paraplegia after aortic surgery. In the 2 cases presented here, however, the orifices of the Adamkiewicz arteries, which were clearly confirmed by anatomic observation at autopsy, were not blocked by the stent graft. Therefore, impairment of blood flow of the Adamkiewicz arteries is not believed to be the cause of postoperative paraplegia. 


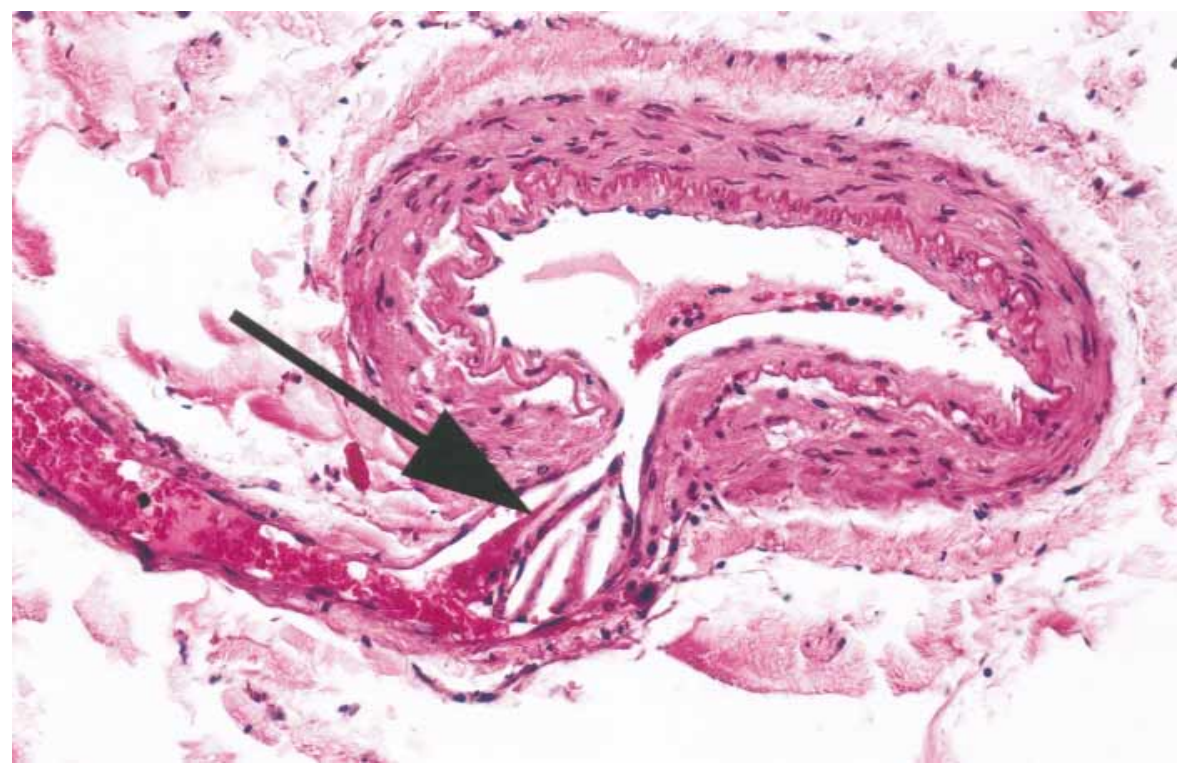

Figure 3. Micrograph of cholesterin embolus (hematoxylin and eosin stain, $\times 400$ ): in stained paraffin sections of the anterior spinal artery at the level of Th6, a clearly recognizable cluster of small cholesterin crystals is visible as white, optically empty, spike-like structures within the vascular lumen (black arrow), which appears to be occluded. The cholesterin embolus is surrounded by fibrin deposits and leukocytes.

Kato and colleagues ${ }^{3}$ reported that among 26 patients with thoracic aortic aneurysms treated with this method, spinal cord injury was observed in only 1 patient who underwent a prolonged circulatory arrest time.

In patient 1 , the circulatory arrest time was rather long (80 minutes at a tympanic temperature of $18^{\circ} \mathrm{C}$ ). However, in our experience this duration of arrest is usually tolerable in patients undergoing conventional aortic surgery. In addition, histologic findings of a very limited area of spinal cord infarction-apart from the level of the Adamkiewicz artery-suggest that there should be some local impairment of spinal cord circulation intraoperatively, although neither thrombus nor embolus was observed in the specimen. In patient 2 , a few cholesterin emboli found in the anterior spinal artery might be at least partly responsible for impaired blood flow to the spinal cord. These cholesterin emboli are believed to have been generated from the atherosclerotic aortic wall during stent-graft delivery. It has been reported that catheterbased stent grafting for aneurysms in the descending aorta is also associated with a substantial incidence of paraplegia and stroke, suggesting a potential hazard associated with insertion of the delivery sheath into the diseased aorta. ${ }^{4,5}$ In patient 2 , however, histologic examination demonstrated diffuse spinal cord infarction continuous from the level of C6 to Th11, suggesting intraoperative global ischemia of the spinal cord. Because of hemodynamic instability, the patient remained hypotensive immediately after bypass, a situation that is reported to be strongly associated with impairment of the spinal cord circulation. ${ }^{6}$

In summary, these case reports demonstrate that paraplegia in patients undergoing open surgery with placement of an endovascular stent graft for aortic arch aneurysm may occur without inter- ruption of blood flow to the Adamkiewicz artery. Every effort should be made to shorten the ischemic time of the spinal cord, to maintain stability of the perioperative hemodynamics, and to avoid generating cholesterin embolus from the atheromatous aorta, which is also a possible cause of spinal cord injury during catheterbased stent grafting.

We thank Prof Dr Reinhard Horvat for his assistance with the manuscript.

\section{References}

1. Kato M, Ohnishi K, Kaneko M, Ueda T, Kishi D, Mizushima T. A new grafting-implanting method for thoracic aortic aneurysm or dissection with a stent-graft. Circulation. 1996;94(Suppl):II-188-93.

2. Kotsuka Y, Takamoto S, Ezure M, Miyairi T, Ono M, Tanaka K, et al. Open surgery combined with stent-graft procedure for an aortic arch aneurysm. Proceedings of 49th International Congress of the European Society for Cardiovascular Surgery, 2000. p. 81-5.

3. Kato M, Kaneko M, Kuratani T, Miyamoto Y, Mitsuno M, Shirakawa $\mathrm{Y}$, et al. Surgical treatment for distal aortic arch aneurysm using stent grafting. J Jpn Surg Soc. 1999;100:486-90.

4. Mitchell RS, Dake MD, Semba CP, Fogarty TJ, Zarins CK, Liddle RP, et al. Endovascular stent-graft repair of thoracic aortic aneurysms. $J$ Thorac Cardiovasc Surg. 1996;111:1054-62.

5. Dake MD, Miller DC, Mitchell RS, Semba CP, Moore KA, Sakai T. The "first generation" of endovascular stent-grafts for patients with aneurysms of the descending thoracic aorta. $J$ Thorac Cardiovasc Surg. 1998;116:689-704.

6. Griepp RB, Ergin MA, Galla JD, Lansman S, Khan N, Quintana C, et al. Looking for the artery of Adamkiewicz: a quest to minimize paraplegia after operations for aneurysms of the descending thoracic and thoracoabdominal aorta. J Thorac Cardiovasc Surg. 1996; 112:1202-15. 